\title{
Dynamical Calling Behavior Experimentally Observed in Japanese Tree Frogs (Hyla japonica)
}

\author{
Ikkyu AIHARA ${ }^{\dagger, \dagger+a)}$, Nonmember, Shunsuke HORAI ${ }^{\dagger \dagger,+\dagger \dagger \dagger}$, Member, Hiroyuki KITAHATA ${ }^{\dagger \dagger}$, Nonmember, \\ Kazuyuki AIHARA ${ }^{\dagger \dagger}, \dagger_{\dagger \dagger}$, Fellow, and Kenichi YOSHIKAWA ${ }^{\dagger \dagger}$, Nonmember
}

\begin{abstract}
SUMMARY We recorded time series data of calls of Japanese tree frogs (Hyla japonica; Nihon-Ama-Gaeru) and examined the dynamics of the experimentally observed data not only through linear time series analysis such as power spectra but also through nonlinear time series analysis such as reconstruction of orbits with delay coordinates and different kinds of recurrence plots, namely the conventional recurrence plot (RP), the isodirectional recurrence plot (IDRP), and the iso-directional neighbors plot (IDNP). The results show that a single frog called nearly periodically, and a pair of frogs called nearly periodically but alternately in almost anti-phase synchronization with little overlap through mutual interaction. The fundamental frequency of the calls of a single frog during the interactive calling between two frogs was smaller than when the same frog first called alone. We also used the recurrence plots to study nonlinear and nonstationary determinism in the transition of the calling behavior. Moreover, we quantified the determinism of the nonlinear and nonstationary dynamics with indices of the ratio $R$ of the number of points in IDNP to that in RP and the percentage $P_{D}$ of contiguous points forming diagonal lines in RP by the recurrence quantification analysis (RQA). Finally, we discuss a possibility of mathematical modeling of the calling behavior and a possible biological meaning of the call alternation.

key words: Japanese tree frogs, calling behavior, synchronization, recurrence plot
\end{abstract}

\section{Introduction}

Synchronization is everywhere in coupled nonlinear systems with oscillations [1]-[3]. In particular, the system of biological oscillators has been studied extensively and intensively, and various kinds of synchronous behavior were reported; for example flashing fireflies and calls of crickets [2]-[4].

In this paper, we analyse calling behavior of Japanese tree frogs (Hyla japonica) (see Fig. 1) especially from the viewpoint of nonlinear dynamics. Japanese tree frogs are distributed ubiquitously and widely from Hokkaido to Yakushima in Japan [5]-[7]. A male Japanese tree frog calls

\section{Manuscript received November 27, 2006.}

Manuscript revised March 30, 2007.

Final manuscript received May 15, 2007.

${ }^{\dagger}$ The author is with the Department of Physics, Faculty of Science, Kyoto University and the Department of Applied Analysis and Complex Dynamical Systems, Graduate School of Informatics, Kyoto University, Kyoto-shi, 606-8501 Japan.

${ }^{\dagger}$ The authors are with the Department of Physics, Graduate School of Science, Kyoto University, Kyoto-shi, 606-8502 Japan.

$t^{\dagger \dagger}$ The authors are with the Aihara Complexity Modelling Project, ERATO, JST, Tokyo, 151-0064 Japan.

${ }^{\dagger+\dagger}$ The authors are with the Institute of Industrial Science, The University of Tokyo, Tokyo, 153-8505 Japan.

a) E-mail: aihara.ikkyu@t02.mbox.media.kyoto-u.ac.jp DOI: 10.1093/ietfec/e90-a.10.2154 and hears sounds, and a pair of them interact in calling behavior, which may be described as a coupled nonlinear system where synchronous behavior can be expected. In fact, there are previous studies on interactions of the frog calls such as interactive calling behavior and entrainment in the meaning of a one-to-one association between stimulus and response in Pseudacris streckeri [8], entrainment by randomly generated calls in Hyla crucifer [9], effects of call alternation in Hyperolius marmoratus [10], Alytes obstetricans [11], and Alytes cisternasii [11], and even binding effects of vocal and visual signals [12]. Although nonlinear phenomena like entrainment and call alternation were considered in these studies, nonlinear time series analysis which has been rapidly progressing [13]-[24] has not yet been well applied to data of calling behavior in frogs. Further, nonlinear dynamics of interactive calling behavior in Japanese tree frogs has been sufficiently analysed neither. Since calling properties of frogs are species-specific [25], it should be worth analysing calling behavior of Japanese tree frogs. In this paper, we experimentally observe calling behavior of Japanese tree frogs, analyse the time series data with both linear and nonlinear analysis methods, and examine existence of deterministic nonlinear dynamics in their calling behavior by the recurrence plots [20]-[24].

\section{Methods}

\subsection{Experiments}

The male Japanese tree frogs used in the experiment were collected from paddy fields (see Fig. 2) in Kyoto, Japan $\left(35.04^{\circ} \mathrm{N}\right.$ and $\left.135.79^{\circ} \mathrm{E}\right)$, in the evening on June 4 th, 2005 , and transported to an experimental place about $100 \mathrm{~m}$ away. The collected frogs were individually housed in small plastic cages containing about $0.5 \mathrm{~cm}$ in depth of water which was brought from the paddy fields where the frogs inhabited. Two plastic cages were separated about $50 \mathrm{~cm}$ away to study interactive calling behavior. Their spontaneous calls were recorded with the video camera (DCR-TRV18, SONY) with the rate of $32 \mathrm{kHz}$ for sounds, which was placed about $60 \mathrm{~cm}-1 \mathrm{~m}$ away from the cages so that both vocal and visual signals can be recorded. The experiment was started immediately after collecting the frogs, and had been performed over the night in a usual room at a constant condition without any feeding. Then, in the morning on June 5th, 2005, the frogs were released in the paddy fields where they were col- 


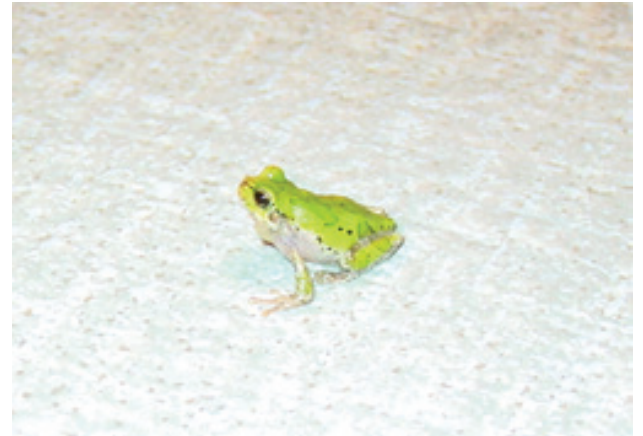

Fig. 1 A male Japanese tree frog (Hyla japonica).

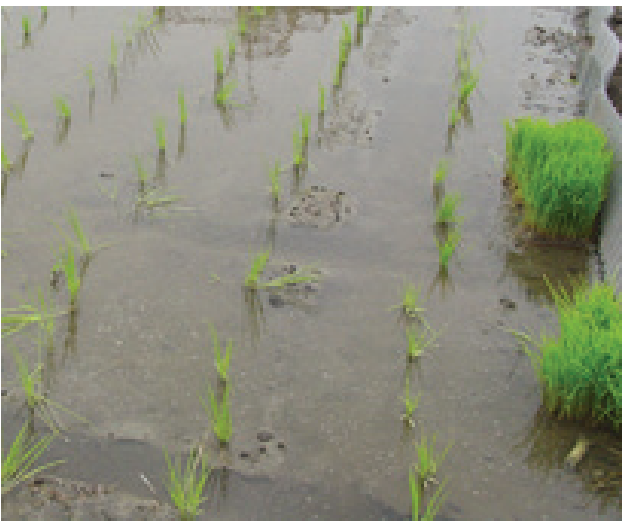

Fig. 2 The paddy field in Kyoto where male Japanese tree frogs were collected.

lected. The temperature and the average moisture at Kyoto were $18.0-29.5^{\circ} \mathrm{C}$ and $68 \%$ on June 4 th and $16.7-24.4^{\circ} \mathrm{C}$ and $62 \%$ on June 5th, 2005.

\subsection{Time Series Analysis}

Time series data of the calls were examined not only with linear time series analysis such as power spectra by Raven Lite 1.0 but also nonlinear time series analysis like reconstruction of orbits with delay coordinates [13]-[19], and the recurrence plot (RP) [20], [21] and its extensions [22], [23], namely the iso-directional recurrence plot (IDRP) and the iso-directional neighbors plot (IDNP).

\subsubsection{Reconstruction of Orbits in a State Space}

Let us denote the observed time series data of calling $\boldsymbol{X}$ as follows:

$$
\boldsymbol{X}=\{X(1), X(2), \ldots, X(L)\},
$$

where $L$ is the total duration of the observation. A vector $\boldsymbol{X}_{i}$ in a reconstructed state space is defined as follows [13][19]:

$$
\boldsymbol{X}_{i}=\{X(i), X(i+\tau), \ldots, X(i+(n-1) \tau)\},
$$

where $\tau$ is the delay for delay coordinates and $n$ is the dimension of the reconstructed state space.
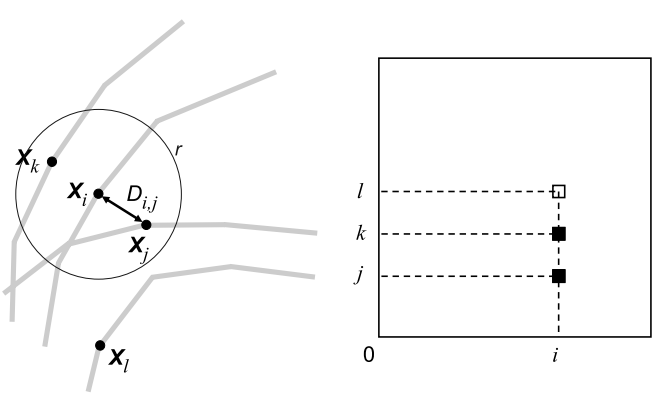

(a)

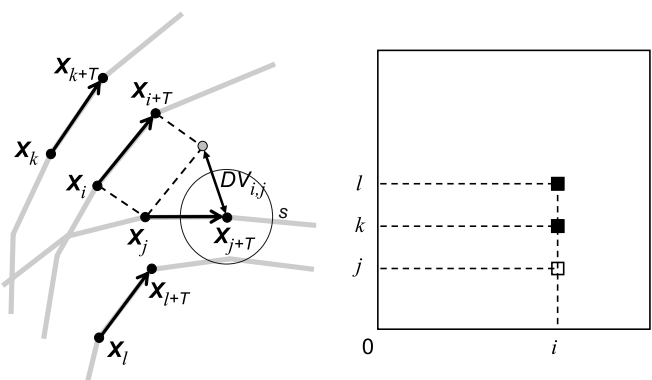

(b)
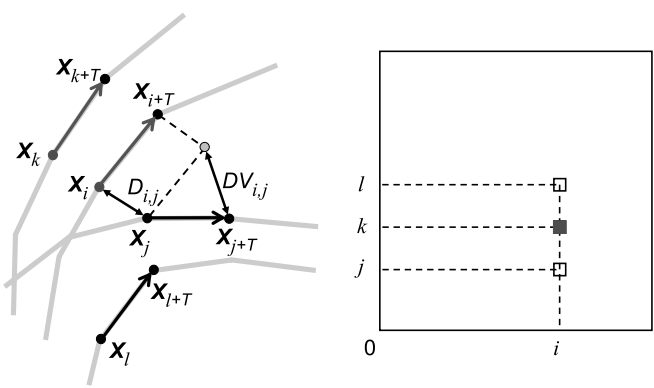

(c)

Fig. 3 Schematic diagrams of (a) the recurrence plot (RP), (b) the isodirectional recurrence plot (IDRP), and (c) the iso-directional neighbors plot (IDNP).

\subsubsection{Recurrence Plots}

The conventional RP is a two-dimensional representation that plots a point $(i, j)$ if the distance $D_{i, j}$ between points $\boldsymbol{X}_{i}$ and $\boldsymbol{X}_{j}$ on a reconstructed orbit is less than or equal to the threshold $r$ as follows [21] (see also Fig. 3(a)):

$$
D_{i, j}=\left\|\boldsymbol{X}_{i}-\boldsymbol{X}_{j}\right\| \leq r,
$$

where $\boldsymbol{X}_{i}$ and $\boldsymbol{X}_{j}$ are vectors in the reconstructed state space. Similarly to the RP, the IDRP characterizes the movement of orbits. That is, a point $(i, j)$ is plotted as shown in Fig. 3(b) if the distance $D V_{i, j}$ is less than or equal to the threshold $s$ as follows [22]:

$$
D V_{i, j}=\left\|\left(\boldsymbol{X}_{i+T}-\boldsymbol{X}_{i}\right)-\left(\boldsymbol{X}_{j+T}-\boldsymbol{X}_{j}\right)\right\| \leq s,
$$

where $T$ is the delay time used for calculation of $D V_{i, j}$ in IDRP, which is set to equal to the reconstruction delay $\tau$ in this paper, and the thresholds $s$ and $r$ are determined so that $10 \%$ of the points except the meaningless ones exactly on 
the diagonal line are plotted in each recurrence plot.

The IDNP is simply the product of RP and IDRP: $I D N P=R P \cap I D R P$ (see also Fig. 3(c)) [22]. Therefore, the IDNP represents the points that are close to each other and are moving in similar directions in the reconstructed state space, which is a very characteristic peculiar to deterministic dynamics. It should be noted that the recurrence plots are useful for analysis of not only nonlinear but also nonstationary dynamics [21], [24] and have much applicability to real-world systems such as the blast furnaces and the genetic sequences [26], [27].

To quantify the determinism from the recurrence plots of RP and IDNP, we calculate the following ratio $R$ of the numbers of points in IDNP to that in RP [22], [23]:

$$
R=\frac{|I D N P|}{|R P|},
$$

where $|R P|$ and $|I D N P|$ are the numbers of points plotted in RP and IDNP, respectively. We also used the percent determinism $P_{D}$ of the recurrence quantification analysis (RQA) [28]-[30] as a measure of determinism. $P_{D}$ represents the percentage of contiguous points forming diagonal lines in RP. The diagonal line segments also reflect deterministic dynamics because they represent similar continuous timeevolutions. Both $R$ and $P_{D}$ have high values in deterministic data [22], [23], [28]-[30].

\section{Experimental Results}

\subsection{Periodicity and Anti-Phase Synchronization}

Figure 4 shows the time series data of calls recorded from (a) a single frog calling alone and from (b) two mutually interacting frogs, where the upper and lower panels in (a) and (b) show the waveforms and the spectrograms, or the sonograms in time durations of $1.8 \mathrm{~s}$, respectively. A single frog called nearly periodically as shown in Fig. 4(a). After this frog called alone for a while, the second frog began to call at about $9.7 \mathrm{~s}$, where the timing of the first call of the second frog is shown by the red arrow in Fig. 4(b); two frogs called alternately in almost anti-phase synchronization with little overlap as shown in the waveform of Fig. 4(b).

Next, we transformed the observed time series data to intensity data, as shown in Fig. 5 where the original amplitude data are squared and averaged over a window of $0.05 \mathrm{~s}$, and re-sampled every $0.01 \mathrm{~s}$. Figure 6 shows the reconstructed orbits of the calls of a single frog and two frogs in delay coordinates [13]-[19]. The orbits of Fig. 6 are reconstructed from the time series data of the intensity of the calls shown in Fig. 5, where the delay time for delay coordinates is 0.03 s. Figures 6(a), (b) and Fig. 6(c) represent the reconstructed orbits of the intensity time series data of a single frog calling alone and that of two frogs calling together through mutual interaction, respectively; Figs. 6(a) and (b) correspond to the starting transient and the almost steady periods in calling of the single frog. Figures 6(b) and (c) show that both the calls of a single frog and those of two interacting frogs are nearly periodic; the latter has roughly two-fold structure. The power spectrum analysis of the time series data showed that the main frequency of the calling by a single frog in Fig. 4(a) and that of the calling by two

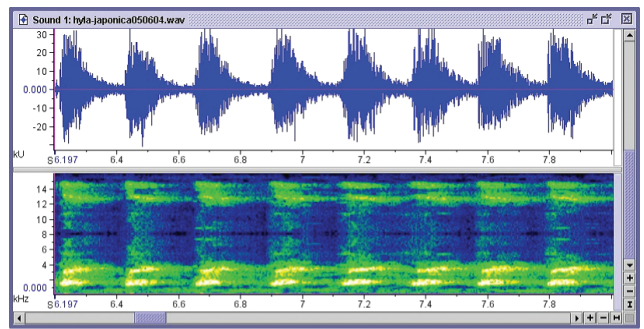

(a)

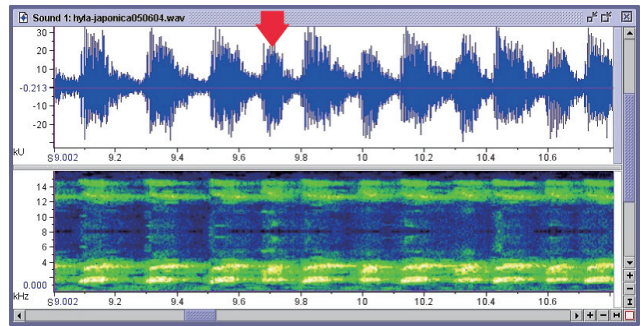

(b)

Fig. 4 The time series data of calls of (a) a single frog calling alone and (b) two mutually interacting frogs. The upper and lower panels in (a) and (b) show the waveforms and the spectrograms, or the sonograms where the abscissa, the ordinate, and the brightness represent the time, the frequency, and the power, respectively. The time intervals are 6.2-8.0 s in (a) and 9.0 $10.8 \mathrm{~s}$ in (b). The red arrow in Fig. 4(b) shows the timing when the second frog began to call.

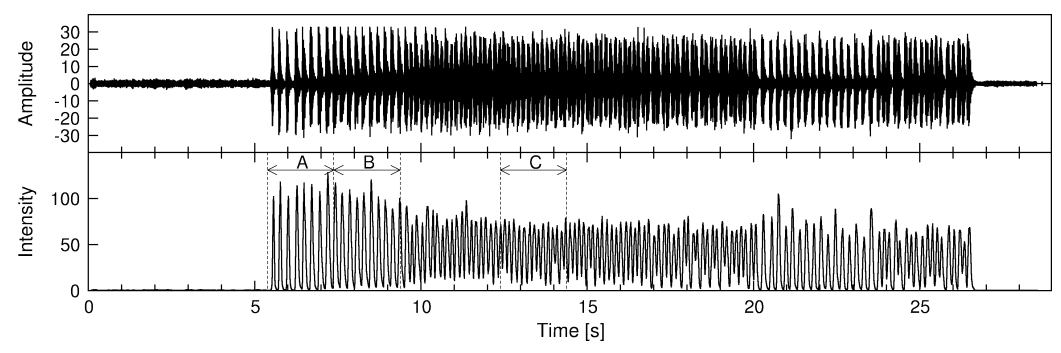

Fig. 5 The transformation of the time series data into intensity data, where the original amplitude data are squared and averaged over a window of $0.05 \mathrm{~s}$, and re-sampled every $0.01 \mathrm{~s}$. The time intervals A, $\mathrm{B}$, and $\mathrm{C}$ correspond to the reconstructed orbits in Figs. 6(a), (b), and (c), respectively. 


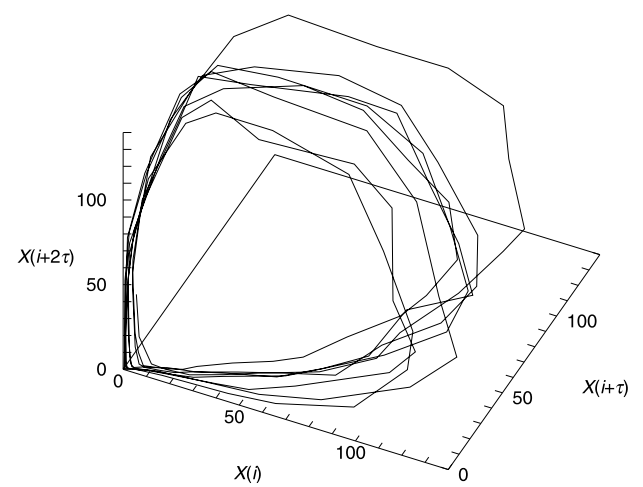

(a)

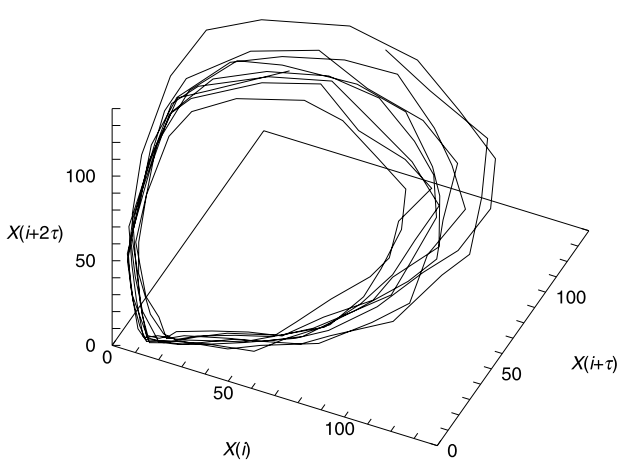

(b)

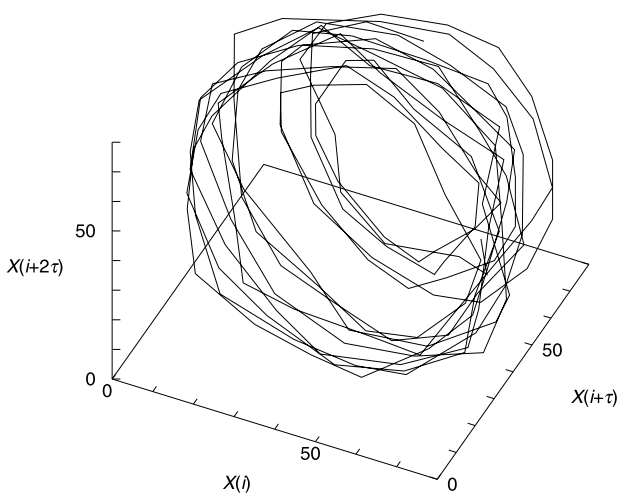

(c)

Fig. 6 Reconstructed orbits in a 3-dimensional space, which are obtained from the time series data of the intensity in Fig. 5 by embedding them with delay coordinates. Figures 6(a), (b), and (c) correspond to the time intervals A, B, and C in Fig. 5, respectively. The delay is set at $0.03 \mathrm{~s}$.

interacting frogs in Fig. 4(b) are about $4.2 \mathrm{~Hz}$ and $6.8 \mathrm{~Hz}$, respectively. The latter value means that each frog was calling with the frequency of about $3.4 \mathrm{~Hz}$ in the mutually interacting behavior. In other words, the fundamental frequency of the calling of the first frog during the interactive calling behavior in Fig. 4(b) is lower than that observed when the same frog first called alone in Fig. 4(a).

3.2 Nonstationary Dynamics with Emergence, Disappearance, and Re-emergence of Anti-Phase Synchronization

Figures 7(a), (b), and (c) show the RP, the IDRP, and the

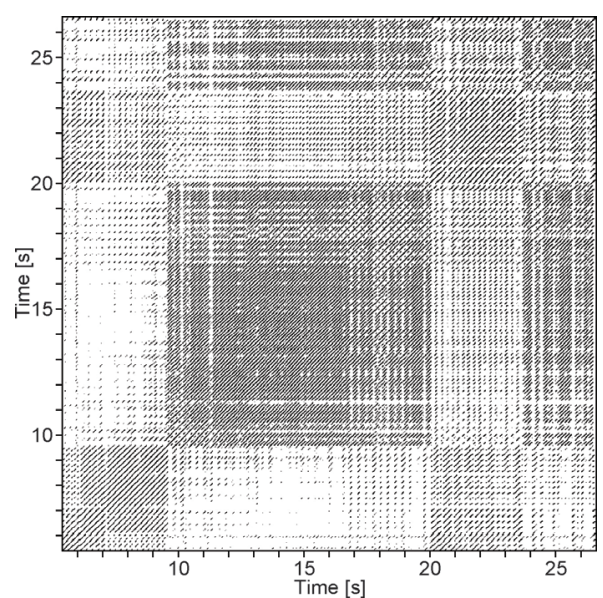

(a)

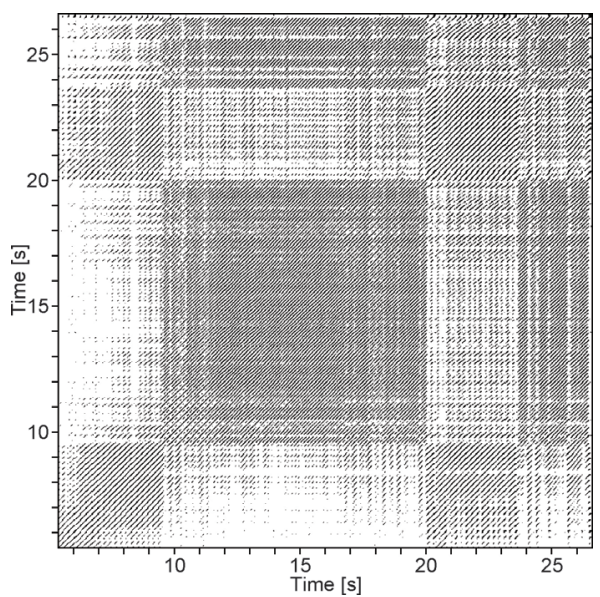

(b)

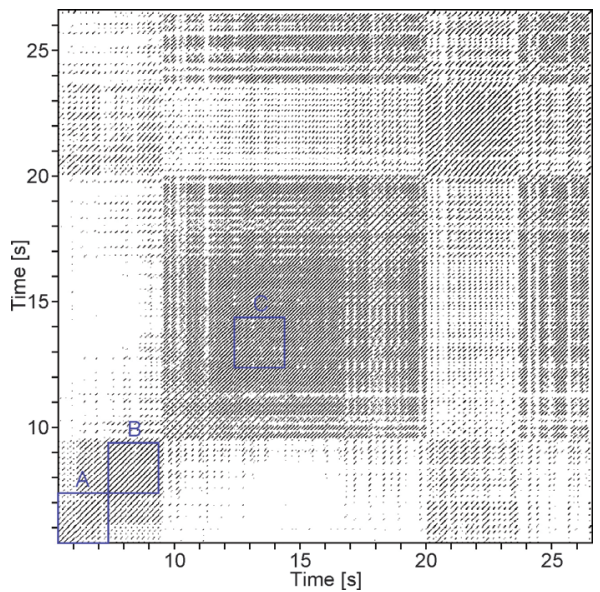

(c)

Fig. 7 (a) the RP [20], (b) the IDRP, and (c) the IDNP [22] of the intensity time series data in the calling behavior with the embedding dimension of 5 and the delay time of $0.03 \mathrm{~s}$. The blue squares A, B, and C in (c) correspond to the plots of the time intervals A, B, and C in Fig. 5, respectively.

IDNP of the intensity time series data in the calling behavior. RP visualizes distances between each pair of state points on reconstructed orbits and also represents stationarity of time series data. In contrast, IDRP characterizes sim- 


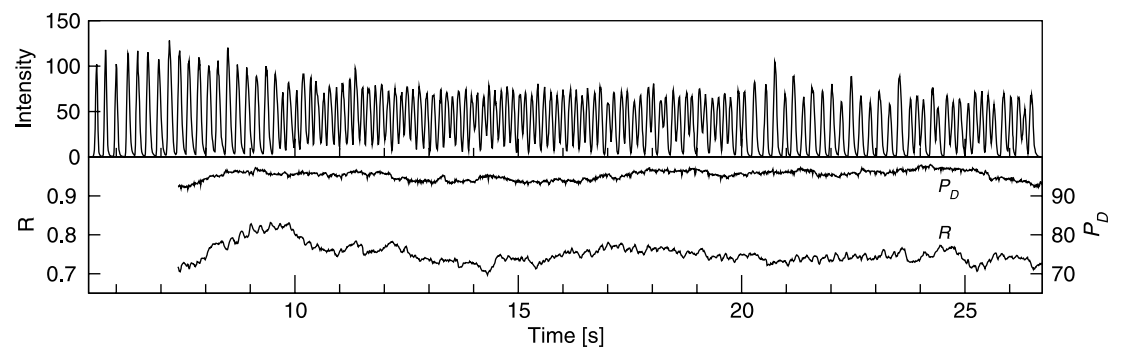

Fig. 8 Temporal changes in the ratio $R$ of the number of points in IDNP to that in RP [22], [23] and the percent determinism $P_{D}$ of RQA [28]-[30], which were analysed with moving time windows of $2 \mathrm{~s}$.

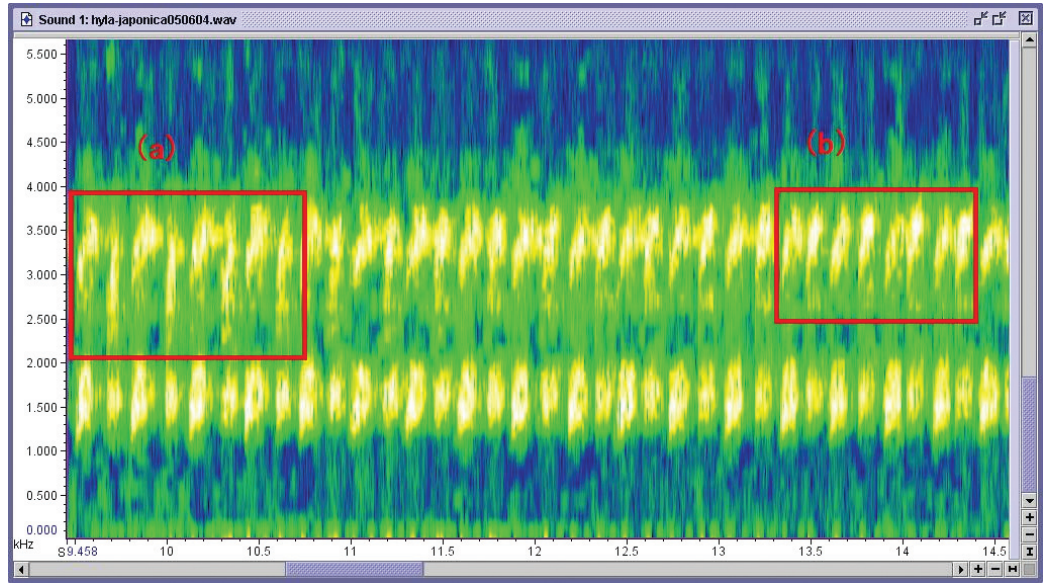

Fig.9 The spectrogram, or the sonogram of the calling data during about 9.5-14.5 s, which shows the shift of tone frequencies around $3.5 \mathrm{kHz}$ during the call alternation, where the abscissa, the ordinate, and the brightness represent the time, the frequency, and the power, respectively.

ilarity of movement directions of reconstructed orbits and can be combined with RP to construct IDNP. Figures 7(a), (b), and (c) show that similar patterns can be observed all in RP, IDRP, and IDNP. This property means that neighboring points move to similar directions in the reconstructed state space, which is a very characteristic peculiar to deterministic systems including chaotic ones [22], [23]. Therefore, Figs. 7(a), (b), and (c) suggest existence of deterministic dynamics in the time series data of Fig. 5 in the calling behavior. A careful observation of patterns along the diagonal direction in Figs. 7(a), (b) and (c) reveals that the first frog called alone until about $9.7 \mathrm{~s}$ as shown by a gray square region at the left-bottom in each of the figures. The region includes the plots of the time intervals $\mathrm{A}$ and $\mathrm{B}$ in Fig. 5, which are indicated by the blue squares in Fig. 7(c). These blue squares A and B correspond to the starting and the almost steady periods of calling of the first frog. After that, the second frog started calling at about $9.7 \mathrm{~s}$ whose timing is indicated by the red arrow in Fig. 4(b), and the two frogs kept calling interactively with nearly anti-phase synchronization, as indicated by a large gray square region at the center of each of Figs. 7(a), (b) and (c). This region includes the plots of the time interval $\mathrm{C}$ in Fig. 5, as indicated by the blue square $\mathrm{C}$ in Fig. 7(c). This anti-phase calling of two frogs stopped at about $20 \mathrm{~s}$, leaving the first frog calling alone again. Then, the interactive calling of two frogs re-started at about $24 \mathrm{~s}$. This transition of the calling behavior is shown by two square regions at the right-top along the diagonal direction in each of Figs. 7(a), (b) and (c). Thus, the recurrence plot analysis in Fig. 7 demonstrates the existence of nonlinear and nonstationary deterministic dynamics with emergence, disappearance, and re-emergence of antiphasely synchronized calling between two frogs through the whole calling period of Fig. 5 from the viewpoint of nonlinear dynamics in the reconstructed state space.

Figure 8 shows the temporal changes in the ratio $R$ of the number of points in IDNP to that in RP [22], [23] and the percent determinism $P_{D}$ of RQA [28]-[30], which were analysed with moving time windows of $2 \mathrm{~s}$. Both of $R$ and $P_{D}$ keep large values throughout the time course, while the calling state changes. These properties also confirm the existence of deterministic dynamics in the calling behavior statistically and quantitatively.

\section{Discussion}

\subsection{Possibility of Mathematical Modeling}

We have confirmed the existence of deterministic dynamics in the calling behavior of two interacting male frogs by the nonlinear time series analysis. In the experiment, a single male frog called nearly periodically and two frogs called 
alternately in almost anti-phase synchronization through interaction. In general systems, when each element behaves periodically with a similar frequency and a pair of such elements interact weakly, the phase reduction theory is widely applicable [1]. It is not difficult to show that the dynamics in the calling behavior of Japanese tree frogs can be qualitatively described with a simple phase oscillator model [1] with the sinusoidal interaction function and phase shift parameters as the first-order approximation of the phase response curve, which can explain both anti-phase synchronization and change of calling periods [31], [32]. There are many future problems, however, for improvement of the mathematical modeling on the calling behavior of Japanese tree frogs as follows:

(1) It is probable that frogs interact not continuously but only when they call. If we modify the coupling strength to be dependent on the timing of the calls of the other frog, the phase model may be more plausible as a model of calling behavior of frogs.

(2) The interaction term can be generally much more complicated [9] than the simple sinusoidal function. It is an important future problem to measure real data of the phase response curves experimentally, and use them to define the interaction function of the calls peculiar to Japanese tree frogs.

(3) The change like adaptation in coupling strength and the difference of intrinsic natural frequencies of two frogs are important for the understanding the dynamics of the coupled system. We need to make an experiment to elucidate such characteristics.

(4) A frog may respond to calls of another frog with a certain time delay [8]. We should explore effects of time delays on the dynamics of the anti-phase synchronization with models of delayed dynamical systems.

(5) The main components in tone frequencies of male Japanese tree frogs are $1.7 \mathrm{kHz}$ and $3.5 \mathrm{kHz}$ [6]. As shown in Fig. 9, during the call alternation, the difference of tone frequencies around $3.5 \mathrm{kHz}$ between two frogs in region (a) was larger than that in region (b). This phenomenon of the tone frequency shift suggests existence of much more complicated dynamics beyond simple phase oscillator models.

\subsection{Possible Biological Meaning}

The calling behavior in a pair of male Japanese tree frogs has two conspicuous characteristics. The first is that the natural frequency of the frog which first called alone was larger than the fundamental frequency of the same frog in the interactive calling, which is in contrast to Hyla crucifer with excitatory effects [9] and the midwife toads which increased calling rates when responded to by a non-overlapping call [11]. It should be also noted, on the other hand, that decrease of calling rates due to interaction was observed in Hyla crucifer when two males approach closely [9] and in Hyla microcephala when a male is interrupted by calls of another frog [33]. The second is that almost anti-phase synchronization with little overlap was observed as shown in Fig. 4(b). This property is different from widely observed call alternation e.g. in Pseudacris streckeri [8] and Hyla crucifer [9] where the second frog calls immediately after the first frog, leaving a relative long interval until the next call of the first frog, which is rather close to in-phase synchronization.

It is said that there are two main purposes in calling behavior of male frogs; namely to attract females and to tell other males their own positions [5]. In the previous study, the role of call alternation was suggested as maintaining distances between male frogs in Hyperolius marmoratus [10]. It is known that male Japanese tree frogs are distributed in bleeding assemblages with a low density [5], which may be understood as the result of maintaining distances through call alternation. It is an interesting future problem to examine spatial distribution of many male Japanese tree frogs in real fields. With respect to the role of attracting females, call alternation could have a role for one male frog to be distinguished by females from the neighboring males. Since there is a negative report to this effect in Hyperolius marmoratus [10], we also need to experimentally examine actual effects of call alternation in Japanese tree frogs. It should be noted that almost anti-phase synchronization in calling behavior of Japanese tree frogs naturally realizes little overlap between calls of two frogs, which may be useful when females select males [11].

\section{Conclusion}

We recorded and analyzed the time series data of calls of Japanese tree frogs (Hyla japonica). The results showed that a single frog called alone nearly periodically, and a pair of frogs called alternately and nearly periodically in almost anti-phase synchronization with little overlap; after one frog called several times alone, the second frog began to call, and the two frogs called alternately. The recurrence plot analysis elucidated the existence of deterministic dynamics in the transition of the calling behavior with emergence, disappearance, and re-emergence of nearly anti-synchronization. The frequency of the calls of the first frog during the interactive calls of two frogs is smaller than that observed when the same frog first called alone.

We discussed a possibility of the mathematical modeling of these phenomena. It is our important future problem to further analyse the calling behavior of Japanese tree frogs (Hyla japonica) like the phase response curve towards mathematical modeling peculiar to Japanese tree frogs. We also discussed a possible biological meaning of the call alternation. The distribution of male Japanese tree frogs with a low density may be understood as the result of call alternation with the effect of maintaining distances between males. It is also an important future problem to examine actual roles of call alternation of Japanese tree frogs in real fields. 


\section{Acknowledgments}

This study was partially supported by a Grant-in-aid for the Scientific Research (No.18GS0421) on "Construction of functional de novo cell" and the 21st Century COE Program of "Center of Excellence for Research and Education on Complex Functional Mechanical Systems" at Kyoto University from the Ministry of Education, Culture, Sports, Science and Technology of Japan.

We'd like to thank Y. Kuramoto, I. Tsuda, H. Kori, H. Fujisaka, M. Thiel, J. Kurth, A. Nagano, and K. Sogo for their stimulating discussions and valuable comments.

\section{References}

[1] Y. Kuramoto, Chemical Oscillations, Waves, and Turbulence, Springer-Verlag, N.Y., 1984.

[2] S.H. Strogatz, Nonlinear Dynamics and Chaos, Perseus Publishing, Cambridge, 1994.

[3] A. Pikovsky, M. Rosenblum, and J. Kurths, Synchronization: A universal concept in nonlinear sciences, Cambridge University Press, Cambridge, 2001.

[4] A.T. Winfree, "Biological rhythms and the behavior of populations of coupled oscillators," J. Theor. Biol., vol.16, pp.15-42, 1967.

[5] M. Matsui, Natural History of the Amphibia, University of Tokyo Press, 1996.

[6] N. Maeda and M. Matsui, Frogs and Toad of Japan: Revised edition, Bun-Ichi Sogo Shuppan, Tokyo, 1999.

[7] T. Matsuhashi and F. Okuyama, Frogs and Toads of Japan + Salamander, Yama-Kei Publishers, Tokyo, 2002.

[8] J.J. Loftus-Hills, “Analysis of an acoustic pacemaker in Strecker's chorus frog, Pseudacris streckeri (Anura:Hylidae)," J. Comp. Physiol., vol.90, pp.75-87, 1974.

[9] R.E. Lemon and J. Struger, "Acoustic entrainment to randomly generated calls by the frog, Hyla crucifer," J. Acoust. Soc. Am., vol.67, no.6, pp.2090-2095, 1980.

[10] N.I. Passmore and S.R. Telford, "The effect of chorus organization on mate localization in the painted reed frog (Hyperolius marmoratus)," Behav. Ecol. Sociobiol., vol.9, pp.291-293, 1981.

[11] J. Bosch and R. Márquez, "Acoustical interference in the advertisement calls of the midwife toads (Alytes obstetricans and Alytes cisternasii)," Behaviour, vol.137, pp.249-263, 2000.

[12] D.B. Kelley, "Vocal communication in frogs," Curr. Opin. Neurobiol., vol.14, pp.751-757, 2004.

[13] F. Takens, "Detecting strange attractors in turbulence," in Dynamical Systems of Turbulence, ed. D.A. Rand and B.S. Young, vol.898 of Lecture Notes in Mathematics, pp.366-381, Springer-Verlag, Berlin, 1981.

[14] T. Sauer, J.A. Yorke, and M. Casdagli, "Embedology," J. Statistical Physics, vol.65, no.3/4, pp.579-616, 1991.

[15] E. Ott, T. Sauer, and J.A. Yorke, eds., Coping with Chaos, John Wiley \& Sons, N.Y., 1994.

[16] A.S. Weigend and N.A. Gershenfeld, eds., Time Series Prediction: Forecasting the Future and Understanding the Past, Addison-Wesley Publishing Company, Reading, 1994.

[17] H.D.I. Abarbanel, Analysis of Observed Chaotic Data, SpringerVerlag, N.Y., 1996.

[18] H. Kantz and T. Schreiber, Nonlinear Time Series Analysis, Cambridge University Press, Cambridge, 1997.

[19] T. Ikeguchi, T. Yamada, and M. Komuro, (ed. K. Aihara), Theory and Applications of Chaos Time Series Analysis, Sangyo-Tosho, Tokyo, 2000.

[20] J.P. Eckmann, S.O. Kamphorst, and D. Ruelle, "Recurrence plots of dynamical systems,” Europhys. Lett., vol.4, no.9, pp.973-977, 1987.
[21] M.C. Casdagli, "Recurrence plots revisited," Physica D, vol.108, pp.12-44, 1997.

[22] S. Horai, T. Yamada, and K. Aihara, "Determinism analysis with isodirectional recurrence plots," T. IEE Japan, vol.122-C, no.1, pp.141147, 2002.

[23] T. Yamada, S. Horai, J. Takahashi, and K. Aihara, "Chaos and complex systems from the viewpoint of engineering," J. Japan Soc. for Fuzzy Theory and Intelligent Informatics, vol.15, no.6, pp.607-616, 2003

[24] T. Yamada and K. Aihara, "Nonstationary Time Series Analysis with Recurrence Plots and Distribution of Inter-Point Distances," IEICE Trans. Fundamentals (Japanese Edition), vol.J82-A, no.7, pp.10161028, July 1999.

[25] A.S. Feng, J.C. Hall, and D.M. Gooler, "Neural basis of sound pattern recognition in anurans," Prog. Neurobiol., vol.34, pp.313-329, 1990

[26] J. Nakagawa and K. Aihara, "Nonstationary analysis of blast furnace by recurrence plot,” IEICE Trans. Fundamentals (Japanese Edition), vol.J87-A, no.10, pp.1303-1309, Oct. 2004.

[27] J. Takahashi, T. Yamada, and K. Aihara, "Chaos time series analysis and its applications," Bulletin of the Iron and Steel Institute of Japan, vol.9, no.6, pp.362-366, 2004.

[28] J.P. Zbilut and C.L. Webber, Jr., "Embeddings and delays as derived from quantification of recurrence plots," Phys. Lett. A, vol.171, pp.199-203, 1992.

[29] C.L. Webber, Jr. and J.P. Zbilut, "Dynamical assessment of physiological systems and states using recurrence plot strategies," J. Appl. Physiol., vol.76, pp.965-973, 1994.

[30] J.P. Zbilut, N. Thomasson, and C.L. Webber, "Recurrence quantification analysis as a tool for nonlinear exploration of nonstationary cardiac signals," Med. Eng. Phys., vol.24, pp.53-60, 2002.

[31] I. Aihara, H. Kitahata, S. Horai, K. Aihara, and K. Yoshikawa, "Synchronization experimentally observed in calling behaviors of Japanese rain frogs (Hyla-japonica)," Proc. 2006 Inter. Symp. on Nonlinear Theory and its Applications, pp.767-770, 2006.

[32] I. Aihara, "Nearly anti-phase synchronization in calling behavior of Japanese rain frogs," Proc. International Workshop on Synchronization: Phenomena and Analysis, pp.71-72, 2006.

[33] J.J. Schwartz, "Male calling behavior, female discrimination and acoustic interference in the Neotropical treefrog Hyla microcephala under realistic acoustic conditions," Behav. Ecol. Sociobiol., vol.32, pp.401-414, 1993.

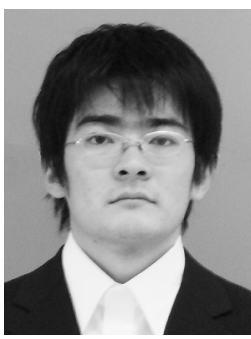

Ikkyu Aihara received the B.E. degree from the Department of Physics, Faculty of Science, Kyoto University in 2006. He is currently a graduate student in the master course at the Department of Applied Analysis and Complex Dynamical Systems, Graduate School of Informatics, Kyoto University. His research interests include experiments and mathematical modeling on synchronous behavior of living things. 


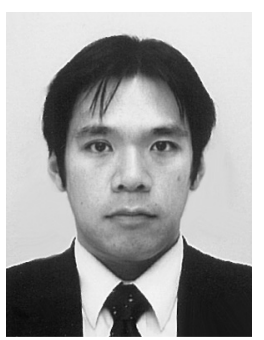

Shunsuke Horai received the B.E. degree from the Department of Electronic Engineering, School of Engineering, Tokyo Denki University, Tokyo, Japan, in 1994, and the Ph.D. degree from the Department of Electronic Engineering, Graduate School of Engineering, Tokyo Denki University, Tokyo, Japan, in 2000. Currently, he is a Researcher of Aihara Complexity Modelling Project, ERATO, Japan Science and Technology Agency (JST). He is also a Cooperative Research Fellow in Institute of Industrial Science, the University of Tokyo. His research interests include nonlinear time series analysis, prediction and visualization of real-world data.

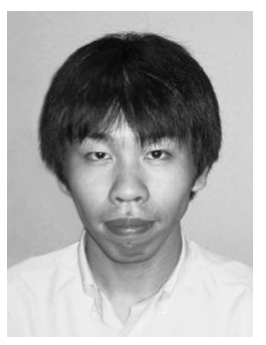

Hiroyuki Kitahata received the B.E. degree in science in 2001 and the Ph.D. degree in science in 2006 from Kyoto University, Kyoto, Japan. Currently, he is Research Associate in Graduate School of Science, Kyoto University. His research interests include experiments and modelling on pattern formation and rhythmic behavior in chemical and biological systems.

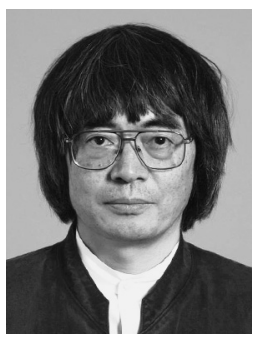

Kazuyuki Aihara received the B.E. degree in electrical engineering in 1977 and the Ph.D. degree in electronic engineering 1982 from the University of Tokyo, Tokyo, Japan. Currently, he is Professor in Institute of Industrial Science and Graduate School of Information Science and Technology, the University of Tokyo. $\mathrm{He}$ is also Director of Aihara Complexity Modelling Project, ERATO, Japan Science and Technology Agency (JST). His research interests include mathematical modeling of biological systems, parallel distributed processing with chaotic neural networks, and time series analysis of complicated data.

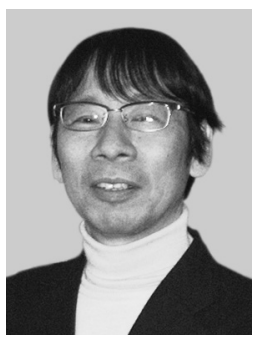

Kenichi Yoshikawa received his Ph.D. on 1977 in Faculty of Engineering from Kyoto University. Currently, he is Professor of Department of Physics, Kyoto University. His major interests concerns with emergence of spatio-temporal structure under thermodynamically open conditions, including the subjects of Nonlinear Physics, Self-Organization in Life, and Folding Transition of Giant DNA. 